\title{
Tabelas de Classificação da Aptidão Física para Frequentadores de Parques Públicos
}

\author{
Physical Aptitude Classification Tables for Users of Public Parks
}

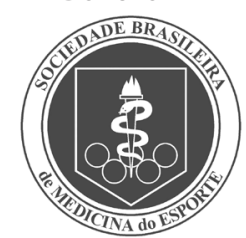

Artigo Original
Júlia Albino

Camila Gobo de Freitas

Victor Matheus da Silva Martins

Hélcio Kanegusuku

Tatiane Prevides Roque

Teresa Bartholomeu

Cláudia Lúcia de Moraes Forjaz

Laboratório de Hemodinâmica da Atividade Motora da Escola de Educação Física e Esporte da Universidade de São Paulo, São Paulo.

Endereço para correspondência: Cláudia Lúcia De Moraes Forjaz Av. Prof. Mello Moraes, 65 - Butantã - 05508-030 - São Paulo, SP Profa. Dra. Cláudia Lúcia de Moraes E-mail: cforjaz@usp.br

\begin{abstract}
Resumo
Introdução: Devido à difusão dos benefícios da atividade física, muitas pessoas passaram a se exercitar em parques públicos, o que incentivou o surgimento de projetos que orientam a prática nestes locais. Estes projetos precisam avaliar a aptidão física de seus clientes com testes fáceis, cujos resultados são comparados a padrões de estadiamento. Porém, os padrões existentes não se adequam à população em questão. Objetivo: Construir tabelas de estadiamento para a avaliação da aptidão física de adultos e idosos. Métodos: Foram utilizados os dados de 713 avaliações feitas em indivíduos de ambos os sexos e com mais de 20 anos. Foram avaliadas a aptidão cardiorrespiratória (marcha estacionária e Cooper), a capacidade muscular (flexão de cotovelo, impulsão vertical e resistência abdominal) e a flexibilidade (sentar e alcançar e flexibilidade de ombros). Resultados: Foi avaliado o percentil 50 de cada teste: marcha estacionária $=105$ passadas; Cooper $=1.200 \mathrm{~m}$; flexão de cotovelo $=20$ rep; resistência abdominal $=18$ rep; força de membros inferiores: $18 \mathrm{~cm}$; flexibilidade de ombros $=1 \mathrm{~cm}$ e flexibilidade lombar $=23 \mathrm{~cm}$. Estes resultados foram maiores que os verificados nas tabelas originais, o que confirmou a necessidade da criação das tabelas específicas. Conclusão: Foram construídas sete tabelas separadas por gênero e faixa etária e com o desempenho classificado em: fraco - < que percentil 20; regular - entre 20 e 40; médio - entre 40 e 60; bom - entre 60 e 80; e ótimo - > que o percentil 80.
\end{abstract}

Palavras-chave: atividade física, aptidão física, adultos.

\begin{abstract}
Introduction: Due to the spread of the health benefits of physical activity, many subjects began to exercise in public parks. This behavior promoted the creation of projects which stimulate and guide on physical activity practice. Nevertheless, for being considered effective, these projects need to evaluate the subjects' physical aptitude by applying easy tests, whose results are compared to classification tables. However, these tables are not adequate to the target population. Objective: To design fitness classification tables based on simple fitness tests applied to adults and elderly subjects. Methods: Data from 713 evaluations conducted with subjects of both genders older than 20 years were analyzed. Cardiorespiratory fitness (stationary gait and Cooper tests), muscle fitness (elbow flexion, vertical jump and abdominal resistance), and flexibility (sit and reach and shoulder flexibility) were evaluated. Results: The 50 percentile for the tests were stationary gait $=105$ steps; Cooper $=$ $1200 \mathrm{~m}$; elbow flexion $=20$ repetitions; abdominal resistance $=18$ repetitions; legs strength $=18 \mathrm{~cm}$; shoulder flexibility $=1 \mathrm{~cm}$; and lumbar flexibility $=23 \mathrm{~cm}$. These results were higher than the original ones described for each test, which confirmed the need for specific classifications tables. Conclusion: Seven tables were designed with the results separated by gender and age group (20 to 80 year-olds divided in 10-year stages). Results were classified as: weak $=<$ than 20 percentile; average $=$ from 20 to 40 percentile; medium = from 40 to 60 percentile; good $=$ from 60 to 80 percentile; and excellent $=>$ than 80 percentile.
\end{abstract}

Keywords: physical activity, fitness, adults.

\section{INTRODUÇÃO}

Os avanços tecnológicos obtidos com a modernização, industrialização e urbanização resultaram em modificações complexas na sociedade contemporânea, das quais destaca-se $\mathrm{o}$ aumento expressivo da vida sedentáriai ${ }^{(1,2)}$. Atualmente, cerca de $80 \%$ da população brasileira é sedentária ${ }^{(3)}$.

Para reverter este quadro, a prática regular de atividades físicas deve ser estimulada, pois ela promove melhora na capacidade, aumentando a capacidade de trabalho e lazer ${ }^{(4)}$. Assim, o exercício aeróbico leva ao aprimoramento da função cardiovascular, os exercícios resistidos resultam na manutenção da massa óssea e no aumento da massa, força e resistência musculares, e os exercícios de flexibilidade aumentam a amplitude de movimento. Todos estes estímulos resultam, portanto, em melhoras expressivas na saúde e qualidade de vida(4).

Diante de tantos benefícios, diversas campanhas públicas ${ }^{(5)}$ têm tentado difundir para a população a importância da atividade física. 
Felizmente, elas têm apresentado resultados positivos, aumentando o grau de conscientização da população sobre o problema. O número de pessoas que têm começado a praticar atividades físicas têm aumentado nos últimos anos ${ }^{(5)}$.

Para a realização dessa prática, no entanto, é necessária a procura de locais adequados. Nos locais com estrutura formal, como academias, clubes e centros educacionais, esportivos e de lazer, a prática é normalmente realizada com a orientação e supervisão direta de profissionais da área de atividade física. Desse modo, nesses locais, os critérios para avaliar, prescrever e acompanhar a atividade já estão bem estruturados e definidos ${ }^{(4)}$. Por outro lado, nos locais sem estrutura formal de orientação, como parques, praças e praias, a prática é feita sem supervisão direta e, normalmente, a orientação não existe ${ }^{(6)}$. Desta forma, não há modelos definitivos de atuação nesses locais.

Entretanto, em função das campanhas citadas anteriormente, o público interessado em praticar atividades físicas nos locais públicos tem crescido expressivamente ${ }^{(6)}$. Diante desta demanda, na última década, vários projetos foram elaborados para orientar essa prática: "Exercício e Coração", "Saúde no Parque", "Gente no Parque", entre outros. Esses projetos, no entanto, foram elaborados sem um modelo formal único de avaliação, prescrição e acompanhamento da atividade, ou seja, cada um segue uma forma específica de atuação. Assim, há a necessidade de, a partir da experiência destes modelos, se elaborar um modelo teórico-prático geral para orientar a implantação deste tipo de projeto em outros locais públicos.

Em relação à avaliação pré-participação, ela deve incluir duas etapas: a avaliação do risco à saúde e a avaliação da aptidão física. A primeira etapa já possui uma série de orientações internacionais(4). Por outro lado, a avaliação da aptidão física dos usuários de locais públicos foi pouco investigada. Esta avaliação é fundamental para orientar a prática em nível adequado ao praticante e para acompanhar a evolução do mesmo. Entretanto, para ser realizada em locais públicos sem estrutura formal para avaliação, é necessário que esta avaliação seja composta por testes simples e de fácil aplicação, que avaliem os principais componentes da aptidão física relacionada à saúde - potência aeróbia, força/resistência muscular e flexibilidade de forma segura e rápida(4).

Diversos testes podem ser utilizados com este intuito ${ }^{(7)}$ e os resultados obtidos são avaliados por comparação com tabelas de estadiamento. Porém, estas tabelas foram construídas com base em populações gerais internacionais, que apresentam características diferentes da população brasileira que frequenta locais públicos. Desta forma, torna-se importante para uma maior precisão a elaboração de tabelas de estadiamento com dados nacionais e de pessoas que frequentam os locais públicos.

Assim, este estudo teve por objetivo elaborar tabelas de estadiamento para testes simples de avaliação de aptidão física relacionada à saúde motora para serem aplicados em usuários brasileiros que frequentam locais públicos. Para tanto, foram utilizados o resultados do banco de dados do projeto "Exercício e Coração", que ocorre no Parque Fernando Costa desde 2000, sob a coordenação da Escola de Educação Física e Esporte da USP.

\section{MÉTODOS}

\section{Amostra}

Foram analisados testes de 713 indivíduos participantes do projeto "Exercício e Coração" entre os anos de 2000 e 2008, sendo 203 homens e 510 mulheres, com idade média de $58 \pm 12$ anos e com diferentes graus de atividade física.
Todos foram questionados sobre a permissão para a utilização dos dados em pesquisas científicas e, quando o voluntário concordou, assinou um termo de consentimento. Este estudo foi aprovado pelo Comitê de Ética da Escola de Educação Física e Esporte da USP.

\section{MÉTODOS}

Os indivíduos realizaram os testes de aptidão física durante uma avaliação previamente agendada. Para esta avaliação, eles compareceram em jejum de 12 horas e sem terem feito atividade física nas 24 horas anteriores. Cada avaliação foi realizada individualmente e incluiu uma entrevista sobre dados pessoais (sexo, idade, ente outros), dados clínicos (presença de sintomas, doenças e fatores de risco cardiovasculares, além do uso regular de medicamentos e da presença de outras doenças) e dados sobre a atividade física praticada. Esta entrevista foi elaborada com base nas diretrizes do Colégio Americano de Medicina Esportiva para a triagem pré-participação ${ }^{(4)}$.

Após o questionário foram feitas medidas de peso (balança portátil) e estatura (estadiômetro simples), sendo então calculado o índice de massa corporal (IMC). Quando o IMC era inferior ou igual a 25, o indivíduo foi classificado como normal; o sobrepeso foi caracterizado quando o IMC ficava entre 25,1 e 29,9; e para que o indivíduo fosse classificado como obeso, seu IMC deveria ser igual ou superior a $30^{(8)}$.

O grau de atividade física foi determinado pelo número de minutos e a intensidade de atividade física praticada em uma semana. $\mathrm{O}$ indivíduo foi classificado como inativo quando não praticava atividade física durante a semana; ativo quando praticava mais de 150 minutos/ semana de atividades moderadas e/ou mais de 60 minutos/semana de atividades intensas; e insuficientemente ativo quando seu nível de prática era inferior aos descritos para os ativos ${ }^{(9)}$.

Após a entrevista e as medidas, os voluntários foram encaminhados para a realização dos testes de aptidão física. No entanto, os indivíduos que apresentaram sintomas sugestivos de doenças cardiovasculares não realizaram nenhum dos testes, os que tinham problemas cardíacos conhecidos não realizaram os testes de aptidão cardiovascular e nem de capacidade muscular, e os que apresentaram problemas osteomusculares não realizaram os testes que implicavam na movimentação da articulação acometida pelo problema.

Os testes de aptidão física utilizados foram: teste de marcha estacionária de dois minutos ${ }^{(10,11)}$, teste de Cooper ${ }^{(12)}$, teste de flexão de cotovelo(10,11), teste de resistência abdominal(13), teste de impulsão vertical(14), teste de flexibilidade de ombros(10,11) e teste de flexibilidade lombar ${ }^{(13)}$.

Brevemente, o teste de marcha estacionária de dois minutos $(10,11)$ consistiu na elevação alternada dos joelhos até a altura média da coxa (medida em centímetros no ponto médio entre o joelho e a crista ilíaca), sendo contado o número máximo de passadas em dois minutos.

O teste de Cooper consistiu em andar ou correr a maior distancia possível em 12 minutos, tentando manter a velocidade constante ${ }^{(12)}$. Ele foi executado em uma pista aberta com 320 metros por volta.

No teste de flexão de cotovelo, foi avaliado o número máximo de movimentos de flexão de cotovelo realizados em 30 segundos, sendo que as mulheres seguraram um peso de dois quilos e os homens um peso de quatro quilos ${ }^{(10,11)}$

No teste de resistência abdominal, o avaliado deitou em decúbito dorsal, com os joelhos flexionados e os pés distanciados na direção dos quadris e seguros pelo avaliador, enquanto os braços ficaram estendidos na lateral do corpo. O avaliado elevou o tronco até aproximadamente 45 (graus) do solo. Foi avaliado o número de repetições do movimento realizadas em 30 segundos $^{(13)}$

No teste de impulsão vertical sem auxílio dos braços ${ }^{(14)}$, o ava- 
liado foi orientado a realizar três saltos consecutivos com os braços elevados. Foi considerada a maior diferença entre a altura alcançada nos saltos e a altura marcada antes do salto.

O teste de flexibilidade de ombros ${ }^{(10,11)}$ consistiu na medida da distância entre os dedos das duas mãos. Os braços foram posicionados nas costas do sujeito, um por cima do ombro e outro por baixo. A medida foi considerada negativa quando os dedos não se encontraram e positiva quando houve sobreposição dos dedos.

O teste de flexibilidade lombar ${ }^{(13)}$ consistiu em flexionar o tronco a frente, deslizando as mãos sobre uma régua sem flexionar os joelhos. Foi considerado o valor mais distante alcançado com as mãos, sendo considerado como 0 (zero) centímetros o local da sola dos pés.

\section{Análise dos dados}

Para a confecção das tabelas de estadiamento, os voluntários foram separados por gêneros (mulheres e homens) e faixa etária (20 a 39, 40 a 49, 50 a 59, 60-69 e 70 ou mais anos). Este estadiamento foi feito pela verificação de percentis e a classificação foi estabelecida em: fraco (< que o percentil 20), regular (entre os percentis 20 e 40), médio (entre os percentis 40 e 60), bom (entre os percentis 60 e 80) e ótimo (acima do percentil 80).

\section{RESULTADOS}

A partir da entrevista foi possível avaliar a amostra estudada. As características gerais dessa amostra estão apresentadas na tabela 1.

A partir da análise exposta anteriormente, foram construídas as tabelas de estadiamento apresentadas nas tabelas 2 a 8.

Tabela 1. Características gerais da amostra.

\begin{tabular}{|c|c|}
\hline Características & Valor \\
\hline $\mathrm{N}$ & 713 \\
\hline Homens & $203(28,5 \%)$ \\
\hline Mulheres & $510(71,5 \%)$ \\
\hline \multicolumn{2}{|l|}{ Faixas etárias } \\
\hline 20-39 anos & $61(8,6 \%)$ \\
\hline 40-49 anos & $101(15 \%)$ \\
\hline $50-59$ anos & $211(29,7 \%)$ \\
\hline 60-69 anos & $214(30,1 \%)$ \\
\hline 70 anos ou mais & $118(16,6 \%)$ \\
\hline \multicolumn{2}{|l|}{ Risco cardiovascular } \\
\hline Sintomas & $175(24,5 \%)$ \\
\hline Doenças cardiovasculares conhecidas & $107(15 \%)$ \\
\hline Diabetes melito & $79(11,1 \%)$ \\
\hline Dislipdemia & $222(31,2 \%)$ \\
\hline Hipertensão & $276(38,8 \%)$ \\
\hline Tabagismo & $46(6,5 \%)$ \\
\hline \multicolumn{2}{|l|}{ Atividade física } \\
\hline Sedentários & $82(11,6 \%)$ \\
\hline Insuficientemente ativos & $167(23,6 \%)$ \\
\hline Ativos & $460(64,9 \%)$ \\
\hline \multicolumn{2}{|l|}{ Obesidade } \\
\hline Normal & $248(35,2 \%)$ \\
\hline Sobrepeso & $304(43,1 \%)$ \\
\hline Obeso & $153(21,7 \%)$ \\
\hline
\end{tabular}

Tabela 2. Classificação dos resultados do teste de marcha estacionária de dois minutos divididos pelo gênero e pela idade. Resultados em número de passadas ( $n=543$ ).

\begin{tabular}{c|c|c|c|c|c}
\hline \multicolumn{6}{c}{ Mulheres } \\
\hline Idades & $20-39$ & $40-49$ & $50-59$ & $60-69$ & $70+$ \\
\hline Fraco & $<90$ & $<92$ & $<90,4$ & $<83$ & $<71$ \\
\hline Regular & $90-99,6$ & $92-106$ & $90,4-101,8$ & $83-95$ & $71-84,2$ \\
\hline Médio & $99,6-114$ & $106-112$ & $101,8-112$ & $95-104,4$ & $84,2-95,4$ \\
\hline Bom & $114-125,2$ & $112-121$ & $112-123,6$ & $104,4-117$ & $95,4-102,4$ \\
\hline Ótimo & $>125,2$ & $>121$ & $>123,6$ & $>117$ & $>102,4$ \\
\hline \multicolumn{7}{c|c|c|}{ Homens } & & \\
\hline Idades & $20-39$ & $40-49$ & $50-59$ & $60-69$ & $70+$ \\
\hline Fraco & $<101,8$ & $<89,4$ & $<105,8$ & $<80$ & $<93,8$ \\
\hline Regular & $101,8-116,8$ & $89,4-111$ & $105,8-116,4$ & $80-99,6$ & $93,8-98,2$ \\
\hline Médio & $116,8-134,4$ & $111-127$ & $116,4-126,8$ & $99,6-112,2$ & $98,2-106$ \\
\hline Bom & $134,4-151,8$ & $127-135$ & $126,8-147,8$ & $112,2-128,6$ & $106-113,8$ \\
\hline Ótimo & $>151,8$ & $>135$ & $>147,8$ & $>128,6$ & $>113,8$ \\
\hline
\end{tabular}

Tabela 3. Classificação dos resultados do teste de Cooper divididos pelo gênero e pela idade. Resultado em metros ( $n=203$ ).

\begin{tabular}{c|c|c|c|c|c}
\hline \multicolumn{5}{c}{ Mulheres } \\
\hline Idades & $20-39$ & $40-49$ & $50-59$ & $60-69$ & $70+$ \\
\hline Fraco & $<1.155$ & $<1.151$ & $<1.115$ & $<1.040$ & $<960$ \\
\hline Regular & $1.155-1.215$ & $1.151-1.208,4$ & $1.115-1.173$ & $1.040-1.147$ & $960-1.050$ \\
\hline Médio & $1.215-1.247$ & $1.208,4-1.285$ & $1.173-1.245$ & $1.147-1.203$ & $1.050-1.103$ \\
\hline Bom & $1.247-1.349$ & $1.285-1.376$ & $1.245-1.335$ & $1.203-1.280$ & $1.103-1.130$ \\
\hline Ótimo & $>1.349$ & $>1.376$ & $>1.335$ & $>1.280$ & $>1.130$ \\
\hline & & & Homens & & \\
\hline Idades & $20-39$ & $40-49$ & $50-59$ & $60-69$ & $70+$ \\
\hline Ruim & - & $<1.252$ & $<1.233,4$ & $<1.051$ & $<1.008$ \\
\hline Regular & - & $1.252-1.411,2$ & $1.233,4-1.326$ & $1.051-1.183,4$ & $1.008-1.093$ \\
\hline Médio & - & $1.411,2-1.555,2$ & $1.326-1.405,2$ & $1.183,4-1.262$ & $1.093-1.245$ \\
\hline Bom & - & $1.555,2-1.708$ & $1.405,2-1.452$ & $1.262-1.304$ & $1.245-1.340$ \\
\hline Ótimo & - & $>1.708$ & $>1.454$ & $>1.304$ & $>1.340$ \\
\hline
\end{tabular}

Tabela 4. Classificação dos resultados do teste de flexão de cotovelo divididos pelo gênero e pela idade. Resultado em número de repetições $(n=642)$.

\begin{tabular}{c|c|c|c|c|c}
\hline \multicolumn{6}{c}{ Mulheres } \\
\hline Idades & $20-39$ & $40-49$ & $50-59$ & $60-69$ & $70+$ \\
\hline Fraco & $<17$ & $<15$ & $<16$ & $<16$ & $<14$ \\
\hline Regular & $17-19,4$ & $15-19$ & $16-18$ & $16-19$ & $14-15$ \\
\hline Médio & $19,4-22$ & $19-22$ & $18-21$ & $19-21$ & $15-18$ \\
\hline Bom & $22-25$ & $22-24,8$ & $21-25$ & $21-24,6$ & $18-21$ \\
\hline Ótimo & $>25$ & $>24,8$ & $>25$ & $>24,6$ & $>21$ \\
\hline \multirow{2}{*}{ Idades } & $20-39$ & $40-49$ & $50-59$ & $60-69$ & $70+$ \\
\hline Fraco & $<16$ & $<15,4$ & $<15$ & $<16$ & $<15$ \\
\hline Regular & $16-18,8$ & $15,4-19$ & $15-18$ & $16-18$ & $15-19,2$ \\
\hline Médio & $18,8-23,2$ & $19-22$ & $18-20$ & $18-21$ & $19,2-20,8$ \\
\hline Bom & $23,2-27,6$ & $22-23,6$ & $20-21$ & $21-24,6$ & $20,8-22,4$ \\
\hline Ótimo & $>27,6$ & $>23,6$ & $>21$ & $>24,6$ & $>22,4$ \\
\hline
\end{tabular}


Tabela 5. Classificação dos resultados do teste de resistência abdominal divididos pelo gênero e pela idade. Resultado em número de repetições ( $n=548$ ).

\begin{tabular}{c|c|c|c|c|c}
\hline \multicolumn{6}{c}{ Mulheres } \\
\hline Idades & $20-39$ & $40-49$ & $50-59$ & $60-69$ & $70+$ \\
\hline Fraco & $<14,6$ & $<13$ & $<13$ & $<12,8$ & $<12$ \\
\hline Regular & $14,6-19$ & $13-15$ & $13-17$ & $12,8-16$ & $12-16$ \\
\hline Médio & $19-21$ & $15-18$ & $17-20$ & $16-19$ & $16-18$ \\
\hline Bom & $21-26$ & $18-23$ & $20-25$ & $19-22,2$ & $18-21,4$ \\
\hline Ótimo & $>26$ & $>23$ & $>25$ & $>22,2$ & $>21,4$ \\
\hline \multirow{2}{*}{ Idades } & $20-39$ & $40-49$ & $50-59$ & $60-69$ & $70+$ \\
\hline Fraco & $<17$ & $<15$ & $<14,2$ & $<14$ & $<11$ \\
\hline Regular & $17-20$ & $15-19$ & $14,2-17,4$ & $14-19$ & $11-17,6$ \\
\hline Médio & $20-25$ & $19-22$ & $17,4-22$ & $19-22,8$ & $17,6-20,4$ \\
\hline Bom & $25-29,6$ & $22-28$ & $22-25$ & $22,8-29$ & $20,4-23,4$ \\
\hline Ótimo & $>29,6$ & $>28$ & $>25$ & $>29$ & $>23,4$ \\
\hline
\end{tabular}

Tabela 6. Classificação dos resultados do teste de impulsão vertical divididos pelo gênero e pela idade. Resultado em centímetros ( $n=595)$.

\begin{tabular}{c|c|c|c|c|c}
\hline \multicolumn{6}{c}{ Mulheres } \\
\hline Idades & $20-39$ & $40-49$ & $50-59$ & $60-69$ & $70+$ \\
\hline Fraco & $<20$ & $<18$ & $<15$ & $<12$ & $<10$ \\
\hline Regular & $20-22$ & $18-21$ & $15-17$ & $12-16$ & $10-11$ \\
\hline Médio & $22-24,7$ & $21-22$ & $17-19$ & $16-17$ & $11-13$ \\
\hline Bom & $24,7-29,2$ & $22-25$ & $19-22$ & $17-20$ & $13-16$ \\
\hline Ótimo & $>29,2$ & $>25$ & $>22$ & $>20$ & $>16$ \\
\hline \multirow{2}{*}{ Idades } & $20-39$ & $40-49$ & $50-59$ & $60-69$ & $70+$ \\
\hline Fraco & $<30,4$ & $<24$ & $<22,4$ & $<20$ & $<13,6$ \\
\hline Regular & $30,4-35$ & $24-29$ & $22,4-25,8$ & $20-22$ & $13,6-17$ \\
\hline Médio & $35-37,2$ & $29-32$ & $25,8-29,2$ & $22-23$ & $17-19$ \\
\hline Bom & $37,2-40,6$ & $32-35$ & $29,2-32$ & $23-25$ & $19-22$ \\
\hline Ótimo & $>40,6$ & $>35$ & $>32$ & $>25$ & $>22$ \\
\hline
\end{tabular}

Tabela 7. Classificação dos resultados do teste de flexibilidade de ombro divididos pelo gênero e pela idade. Resultado em centímetros $(n=654)$.

\begin{tabular}{c|c|c|c|c|c}
\hline \multicolumn{6}{c}{ Mulheres } \\
\hline Idades & $20-39$ & $40-49$ & $50-59$ & $60-69$ & $70+$ \\
\hline Fraco & $<1,4$ & $<(-4)$ & $<(-6)$ & $<(-6)$ & $<(-11)$ \\
\hline Regular & $1,4-3,8$ & $(-4)-1,8$ & $(-6)-(-0,2)$ & $(-6)-(-1)$ & $(-11)-(-5,6)$ \\
\hline Médio & $3,8-7$ & $1,8-4$ & $(-0,2)-2$ & $(-1)-1,2$ & $(-5,6)-0,6$ \\
\hline Bom & $7-9$ & $4-7$ & $2-5$ & $1,2-4$ & $0,6-4$ \\
\hline Ótimo & $>9$ & $>7$ & $>5$ & $>4$ & $>4$ \\
\hline \multirow{2}{*}{ Idades } & $20-39$ & $40-49$ & $50-59$ & $60-69$ & $70+$ \\
\hline Fraco & $<(-8)$ & $<(-7)$ & $<(-7,2)$ & $<(-12,4)$ & $<(-13)$ \\
\hline Regular & $(-8)-5$ & $(-7)-1,2$ & $(-7,2)-(-3)$ & $(-12,4)-(-7)$ & $(-13)-(-7)$ \\
\hline Médio & $5-9$ & $1,2-4$ & $(-3)-4$ & $(-7)-(-2)$ & $(-7)-(-2)$ \\
\hline Bom & $9-11,6$ & $4-8$ & 4 a 10 & $(-2)-5,2$ & $(-2)-5$ \\
\hline Ótimo & $>11,6$ & $>8$ & $>10$ & $>5,2$ & $>5$ \\
\hline
\end{tabular}

Tabela 8. Classificação dos resultados do teste de sentar e alcançar divididos pelo gênero e pela idade. Resultado em centímetros $(n=639)$.

\begin{tabular}{|c|c|c|c|c|c|}
\hline \multicolumn{6}{|c|}{ Mulheres } \\
\hline Idades & $20-39$ & $40-49$ & $50-59$ & $60-69$ & $70+$ \\
\hline Fraco & $<20,4$ & $<18,4$ & $<18$ & $<16$ & $<15$ \\
\hline Regular & $20,4-25,8$ & $18,4-22,8$ & $18-23$ & $16-22$ & $15-20$ \\
\hline Médio & $25,8-28,2$ & $22,8-28$ & $23-29$ & $22-28,4$ & $20-25$ \\
\hline Bom & $28,2-32,6$ & $28-36$ & $29-33$ & $28,4-34$ & $25-32$ \\
\hline Ótimo & $>32,6$ & $>36$ & $>33$ & $>34$ & $>32$ \\
\hline \multicolumn{7}{|c|}{} & & Homens & & \\
\hline Idades & $20-39$ & $40-49$ & $50-59$ & $60-69$ & $70+$ \\
\hline Ruim & $<20$ & $<12$ & $<11$ & $<6,6$ & $<7$ \\
\hline Regular & $20-27,6$ & $12-18$ & $11-17$ & $6,6-13,4$ & $7-10$ \\
\hline Médio & $27,6-33,2$ & $18-23,8$ & $17-20$ & $13,4-17,6$ & $10-16$ \\
\hline Bom & $33,2-39,6$ & $23,8-29$ & $20-25$ & $17,6-26$ & $16-22$ \\
\hline Ótimo & $>39,6$ & $>29$ & $>25$ & $>26$ & $>22$ \\
\hline
\end{tabular}

\section{DISCUSSÃO}

O principal resultado deste estudo foi a elaboração de tabelas de estadiamento por gênero e idade dos resultados de sete testes simples para a avaliação da aptidão física relacionada à saúde adequadas para indivíduos frequentadores de locais públicos.

A elaboração de tabelas com a divisão em gênero e idades é a forma usualmente utilizada para a avaliação da aptidão física. Comparando-se as tabelas obtidas neste estudo com as recomendadas para cada um dos testes, verificam-se diferenças importantes.

O teste de marcha estacionária de dois minutos, originalmente descrito por Rilki e Jones ${ }^{(10,11)}$, possui uma tabela de classificação dividida em gênero e faixas etárias (60-64 anos/65-69 anos/70-74 anos/75-79 anos/80-84 anos/85-89 anos/90-94 anos) baseada em dados de indivíduos americanos idosos. Comparando-se os resultados obtidos por Rilki e Jones ${ }^{(10,11)}$ com os do presente estudo, observamos diferenças em diversos resultados: o percentil 50 para mulheres de 60-64 anos $=91$ vs. 107 passadas, mulheres de $65-69$ anos $=90$ vs. 99 passadas; para os homens, na faixa etária de 60-64 anos $=101$ vs. 105, 65-69 anos $=101$ vs. 103,5, respectivamente. Os resultados obtidos na nossa população foram, portanto, maiores que os observados na população de Rilki e Jones.

Em relação ao teste de Cooper, comparando-se os resultados da tabela original(12) com as deste estudo, observamos que em mulheres de 40 a 49 anos, os resultados originais estão entre 1.200 e 2.350 metros e, neste estudo, entre 1.151 e 1.376 metros. Para homens da mesma faixa etária, os resultados originais vão de 1.350 a 2.500 metros e no presente estudo, de 1.253 a 1.708 metros. Diferenças semelhantes são observadas para outras faixas etárias, ou seja, os valores do presente estudo são inferiores aos observados na tabela original, principalmente para os graus mais elevados de pontuação. Cabe ressaltar, no entanto, que como o teste de Cooper foi incluído na bateria de avaliação do projeto "Exercício e Coração" apenas em 2006, o número de indivíduos que fez este teste foi inferior ao número de participantes nos demais testes, o que resultou na ausência de homens na faixa etária de 20 a 39 anos nesta tabela e pode ter influenciado na classificação geral deste teste.

O teste de flexão de cotovelo, descrito por Rilki e Jones ${ }^{(10,11)}$, também se baseia na população americana e de idosos. Quando comparamos a tabela original com os resultados deste estudo, observamos, respectivamente, que o percentil 50 para mulheres de 60-64 anos foi de 
16 vs. 20,5 repetições, para mulheres de 65-69 anos $=15$ vs. 21 repetições; para homens de 60-64 anos $=19$ vs. 20 repetições e de $65-69$ anos $=18$ vs. 18 repetições. Novamente, estes dados demonstram diferenças entre as populações, principalmente nas mulheres, obtendo-se valores mais elevados nas avaliadas no presente estudo.

O teste de resistência muscular abdominal é amplamente utilizado na literatura. Comparando-se a tabela original de classificação(13) $\mathrm{com}$ a obtida neste estudo, para mulheres entre 40 e 49 anos os resultados vão de três a 25 repetições vs. 13 a 25 repetições, mulheres entre 50 e 59 anos = cinco a 25 repetições vs. 12,8 a 22 repetições e mulheres de 60 a 69 anos = duas a 25 repetições vs. 12 a 21 repetições. Da mesma maneira, para os homens de 40 a 49 anos os resultados variam de cinco a 25 repetições vs. 14 a 25 repetições, para homens de 50 a 59 anos $=$ sete a 25 repetições vs. 14 a 29 repetições e de 60 a 69 anos $=$ cinco a 25 repetições vs. 11 a 23 repetições. Observa-se novamente a diferença e a necessidade de tabelas específicas.

Comparando-se os resultados de estadiamento original do teste de impulsão vertical ${ }^{(14)} \mathrm{com}$ os obtidos no presente estudo, nas mulheres acima de 20 anos, o percentil $50=8$ vs. $18 \mathrm{~cm}$ e nos homens acima de 20 anos o percentil $50=16$ vs. $25 \mathrm{~cm}$, respectivamente. Novamente, o estudo atual apresentou resultados superiores aos originais.

O teste de flexibilidade de ombros também foi proposto por Rilki e Jones ${ }^{(10,11)}$ e as tabelas de estadiamento foram feitas com a população americana idosa. Quando comparamos a tabela original com a deste estudo, observamos, respectivamente para o percentil 50 das mulheres de $60-64$ anos $=-0,5$ vs. $1 \mathrm{~cm}$, de $65-69$ anos $=-1$ vs. $0 \mathrm{~cm}$ e nos homens de $60-64$ anos $=-3,5$ vs. $-0,5 \mathrm{~cm}$ e de $65-69$ anos $=-4$ vs. $-5 \mathrm{~cm}$, o que demonstra que a população deste estudo é mais flexível.

Ao compararmos os resultados da tabela proposta para o teste de sentar e alcançar com a obtida neste trabalho observamos, respectivamente, que as diferenças para o percentil 50 nas mulheres de 40 a 49 anos foram de $28 \mathrm{~cm}$ vs. $24,5 \mathrm{~cm}$ e nas de 50 a 59 anos 27 vs. $27 \mathrm{~cm}$. Da mesma forma, nos homens, verifica-se para o percentil 50, de 40 a 49 anos, 22 vs. $22 \mathrm{~cm}$ e nas de 50 a 59 anos 22 vs. $18 \mathrm{~cm}$. Dessa forma, para este teste os resultados obtidos em nossa população foram bastante semelhantes aos obtidos na população utilizada para a elaboração da tabela original.

Dessa maneira, para a maior parte das variáveis, os valores obtidos na presente investigação foram maiores que os verificados nas tabelas originais. Esta diferença pode ser parcialmente explicada pelo fato da população analisada (frequentadores de parques públicos) apresentar uma prevalência de pessoas ativas $(64,9 \%)$ substancialmente mais elevada do que o que se observa na população geral brasileira - 69 a $80 \%{ }^{(3,15)}$ ou americana 70\%(16). É sabido que indivíduos ativos apresentam melhor aptidão física em todas as variáveis consideradas: aptidão aeróbia, força e resistência musculares e flexibilidade.

\section{CONCLUSÃO}

Diante dos resultados obtidos, é possível concluir que as diferenças observadas entre as tabelas originais e as específicas construídas para a população deste estudo justificam a necessidade de elaboração destas tabelas, como proposto por este trabalho. As tabelas construídas são mais adequadas para a população brasileira que frequenta locais públicos para se exercitar. Desta forma, sugere-se que estes testes e as tabelas construídas neste estudo sejam empregados em programas de orientação de prática saudável de atividades físicas em locais públicos.

\section{AGRADECIMENTOS}

Os autores gostariam de agradecer aos voluntários que participaram deste estudo, à Administração do Parque Fernando Costa e à Associação de Amigos e Ambientalistas do Parque da Água Branca. Este trabalho recebeu apoio financeiro do CNPq (Bolsa IC) e da Pró-Reitoria de Cultura e Extensão da Universidade de São Paulo.

Todos os autores declararam não haver qualquer potencial conflito de interesses referente a este artigo.

\section{REFERÊNCIAS}

1. MInistério da Saúde. Agita Brasil: guia para agentes multiplicadores. In: Saúde Md, editor; 2001.

2. World Health Organization. World health day 2002. Sedentary life style: a global health problem. 2002 [updated 2002; cited 2008]; Available from: http://www.who.int/world-heathy-day/.

3. Monteiro CA, Conde WL, Matsudo SM, Matsudo VR, Bonseñor IM, Lotufo PA. A descriptive epidemiology of leisure-time physical activity in Brazil, 1996-1997. Am J Public Health 2003;14:246-54.

4. American College of Sports Medicine. ACSM's Guidelines for Exercise Testing and Prescription. Philadelphia: Lippincott Williams and Wilkins; 2006.

5. CELAFISCS. Agita São Paulo SP Proposta. 2008 [updated 2008; cited 2008]; Available from: http:// www.agitasp.br/proposta.asp.

6. Torrens LGS. Atuação do profissional de educação física em parques. R Bras Ci e Mov. 2003;11:41-4.

7. Heyward VH. Avaliação Física e Prescrição de Exercício. 4 ed. Porto Alegre: Artmed; 2004.

8. World Health Organization. Diet, Nutrition and the Prevention of Chronic Disease. Geneva; 2003. Report No.: 916 Contract No.: Document Number|.

9. Hallal PC, Victora CG, Wells JC, Lima RC. Physical inactivity: prevalence and associated variables in Brazilian adults. Med Sci Sports Exerc 2003;35:1894-900.

10. Rilki RE, Jones CJ. Functional fitness normative scores for community-residing older adults, ages 60-94. J Aging Phys Act 1999;7:162-81.

11. Rilki RE, Jones CJ. Development and validation of a functional fitness test for community-residing older adults. J Aging Phys Act 1999;7:129-61.

12. Cooper KH. Capacidade Aeróbia. 2 ed. Forum, editor:. Forum; 1972.

13. Canadian Society for Exercise Physiology. The Canadian Physical Activity, Fitness \& Lifestyle Approach: CSEP - Health-Related Appraisal \& Counseling Strategy. In: Physiology CSfE, editor. 3 ed: Canadian Society for Exercise Physiology; 2003.

14. Soares JS, M. Medidas de força muscular. In: CELAFISCS, editor. Testes em ciências do esporte. 5 ed. São Caetano do Sul: CELAFISCS; 1995. p. 73-7.

15. Rego RA, Berardo FA, Rodrigues SS, Oliveira ZM, Oliverira MB, Vasconcellos C, et al. [Risk factors for chronic non-communicable diseases: a domiciliary survey in the municipality of Sao Paulo, SP (Brazil). Methodology and preliminary results]. Rev Saude Publica 1990;24:277-85.

16. Centers for Disease Control. Coronary heart disease attributable to sedentary lifestyle--selected states, 1988. JAMA 1990;264:1390, 1392. 